\title{
Editorial
}

\section{The Batista procedure}

Until recently refractory congestive heart failure under optimal medical treatment could only be treated surgically by heart transplantation. Many factors, such as a lack of donors, an operative mortality rate of $10-20 \%$, and side effects associated with immunosuppression, have prompted heart surgeons to find alternative treatments. Moreover the number of heart failure patients is steadily growing. Although medical treatment has changed the course of the disease, it has only helped to slow the process of deterioration. Patients referred for surgery show a longer evolution than in the past and are probably more sick. Since the early '90s many surgical options have appeared and, like medical treatment, should allow a tailored surgical approach. Ischaemic cardiomyopathies can benefit from myocardial revascularisation even in patients without angina and an ejection fraction below 20\%. ${ }^{2}$ Such patients could already have some kind of ventricular reduction with use of the Dor procedure in dyskinetic and akinetic areas. ${ }^{3}$ More recently, Bolling has called our attention to the deleterious effect of mitral regurgitation in such failing ventricles and advocated a simple annuloplasty to make the mitral valve competent again. ${ }^{4}$ All these options can be used either in isolation or in combination in any given patient. On the other hand, dilated cardiomyopathy has lacked alternative solutions to heart transplantation. Recently, two surgical options became available at the same time: isolated mitral valve repair, and left ventricular reduction with correction of mitral regurgitation - the Batista procedure.

Mitral valve repair was pioneered by Bolling and represents probably the easiest and most elegant way to treat a key factor of congestive heart failure. ${ }^{4}$ In a series of more than 60 patients evenly distributed among ischaemic and dilated cardiomyopathies, Bolling's operative mortality is extremely low $(<2 \%)$. Actuarial survival reaches $82 \%$ at one year and $72 \%$ at two years. More interestingly, all patients have improved not only functionally but also haemodynamically. However, it seems doubtful whether all patients - irrespective of their aetiology, size of left ventricular cavity, or presence of severe pulmonary hypertensioncan benefit from a simple mitral valve repair. It always seems difficult to admit that a procedure has no contraindications and that its promoter did not find any algorithm to make the decision whether or not to correct mitral regurgitation in end stage congestive heart failure patients. No other series has shown similar data. Although results from other authors would show restrictive indications, higher operative mortality and less improvement, the question remains: should this surgical option be thrown away? Probably not, because we are only searching for a palliative procedure which can significantly improve survival and-moderately, if not mildly - functional and haemodynamic status.

The other surgical option - the Batista procedure-has been associated with many mistakes, mainly because of its revolutionary concept and its incredible media impact; both never do well in the medical field. Batista has proposed this operation in a huge country where heart transplantation is not available and therefore no selection criteria was described, no pre- and postoperative data were recorded, and midterm survival could not be accurately reported. Many surgical centres around the world, mainly in the USA, have applied Batista's principles without any critical analysis before its clinical application.

\section{Is patient selection the key?}

Not surprisingly, results were very poor and this option was recommended to be abandoned. However, very few have tried to select patients such as was done at the Cleveland Clinic Foundation, where McCarthy and colleagues have separated ischaemic cardiomyopathies from dilated idiopathic cardiomyopathies. ${ }^{5}$ Considering that a scarry, patchy myocardium could not offer a predictable response to the Batista operation, they have recommended to use it only in idiopathic cardiomyopathies. This tremendous improvement in patient selection has led to improved results. Experience of operating on 57 patients has shown that mechanical support may be necessary to decrease operative mortality as 11 patients $(17 \%)$ required a left ventricular assist device (LVAD) postoperatively. However, not all countries can afford such a policy for economical reasons. Actuarial survival is $82 \%$ at one year and data are not available beyond that period. Young age seems to be a risk factor and this procedure should probably be offered to patients who cannot receive heart transplantation, either for age reasons or for risk factors associated with heart transplantation. Finally, actuarial freedom from failure was close to $60 \%$ at one year. This last point is probably the most crucial because it shows that in this series, left ventricular reduction to a certain extent has unpredictable results. Do these results mean that this operation is not effective and unreliable, or does it mean that selection of candidates is still not restrictive enough? Many other factors concerning the adequate amount of myocardium to be removed, and the need for mitral regurgitation correction (either with mitral repair or with mitral valve replacement), still need to be further analysed and explained. Likewise the whole concept relies on systolic dysfunction, and no one has ever addressed the question of diastolic dysfunction, which can become important when the size of the cavity becomes smaller.

\section{Timing of the procedure}

Many factors concerning indications, timing, and surgical approach need to be clarified. For example, what is the most appropriate timing for such a procedure? Should patients be operated upon when stabilised in New York Heart Association (NYHA) functional class III, or should it be performed when patients are in class IV and sometimes inotrope dependent? In our small series, one patient had been assessed and found to be an excellent candidate. This patient remained stable in NYHA class III for three years. He progressively deteriorated and when he became class IV, specific echocardiographic parameters 
favouring the Batista procedure disappeared. His ongoing deterioration prompted us to insert an LVAD as a bridge to transplantation. We strongly believe that fibrosis may be related to the duration of the disease.

Considering the size and the shape of ventricular reduction, the Laplace law has been applied to this concept. However, it can only be an approximation which does not allow to us to predict scientifically whether removing a width of $3 \mathrm{~cm}$ would be better than removing more or less. Moreover there is no scientific means by which to decide what amount of ventricular mass should be left according to a given size of ventricular cavity. From the surgical standpoint it seems easier to remove a wedge shaped piece of myocardium with its base at the apex of the heart. However, the base is always more dilated than the apex and probably a wedge resection with its base at the base of the heart would be more efficient, although more challenging. Concerning the mitral valve treatment, it remains a key point for those who both favour and deny the efficacy of the Batista procedure. For those who dislike the procedure, an isolated mitral valve repair would always be sufficient, irrespective of the size of the left ventricle, such as in Bolling's theory. For those who favour the Batista operation, we believe that mitral regurgitation correction is only one component of the operation, but is not enough by itself.

We, like McCarthy, have performed the Batista operation in patients with a very dilated left ventricle (end diastolic dimensions $\geqslant 90 \mathrm{~mm}$ ) without concomitant mitral regurgitation, which proved to be successful. This shows that the mitral component in some patients is not the main factor for heart failure. Like others, we believe that papillary muscles, as well as the mitral annulus, are the main components of the left ventricle and play a role, not only in systolic but also in diastolic dysfunction. As opposed to Batista's recommendation which states, "the more myocardium removed, the better", we think that mitral valve repair is probably the only option to treat mitral regurgitation in such patients. Batista's recommendations for resecting more myocardium with the mitral valve apparatus can be detrimental and might explain the poor results reported after such a procedure. Do these results, which do not meet the expectations but still achieve more than $80 \%$ survival at one year, mean that this operation should be abandoned, or does it mean that selection was not adequate? This procedure is not favoured anymore, especially in the USA, because its results were not predictable. Is it because the concept is not valid, or is it because more powerful predictive factors have not been described until now?

\section{Personal series}

In our personal series, we have assessed 52 patients showing idiopathic dilated cardiomyopathies exclusively. Patients, as a first step, met the Cleveland Clinic criteria such as an end diastolic diameter $>70 \mathrm{~mm}$, a wall thickness $>0.7 \mathrm{~cm}$, and a distance between both papillary muscles $>3 \mathrm{~cm}$. As a second step all patients underwent stress dobutamine echocardiography at low doses $(<12 \mu \mathrm{g} / \mathrm{kg} /$ min). Many parameters were recorded before and after dobutamine infusion-mainly stroke volume, wall thickness, mitral regurgitation evolution, and pulmonary pressure. All parameters were searching for a contractile reserve. The most powerful predictive parameter for good outcome seems to be an increase of more than $30 \%$ of the stroke volume provided that pulmonary pressure does not show a steep augmentation. Only eight patients met our criteria and underwent a left ventricular reduction associated with a mitral valve repair. In all instances a prosthetic ring was sutured and an Alfieri stitch used to increase coaptation. It also has to be stressed that in seven cases a tricuspid repair has been performed by using a tricuspid ring. We strongly believe that such correction can decrease right ventricular afterload, just as mitral regurgitation correction decreases left ventricular afterload. Six patients are long term survivors with follow ups of 1-3 years. All have improved significantly - both functionally and haemodynamically - and their left ventricular cavity has not redilated. Of the two deaths, one was caused by septic shock in the early postoperative phase, and the other resulted from chronic renal failure. All patients who underwent this procedure were in NYHA class IV, and four out of eight were inotrope dependent. All patients had a peak oxygen consumption $<14 \mathrm{ml} / \mathrm{min} / \mathrm{m}^{2}$.

Although our experience is very small, we believe that there is a place for the Batista operation in patients with severe congestive heart failure and who, for some reason, cannot undergo heart transplantation. We also believe that no more than $20 \%$ of idiopathic cardiomyopathies are appropriate for this procedure, and that further investigations are required to define a suitable candidate in this regard. According to our histological data, patients who underwent the Batista operation showed mild myocardial fibrosis or none at all. On the other hand, those patients who underwent heart transplantation, after a Batista assessment, showed in all cases diffuse and moderate or severe fibrosis in the myocardium. Is fibrosis the key answer?

\section{The future for the Batista procedure}

Is there any future for this operation? We believe that there is, mainly because of the lack of donors for transplantation and the increasing number of heart failure patients who sooner or later will not respond to optimal medical treatment. Techniques to assist a failing heart in order to allow its potential recovery have recently been proposed. This approach, although uncommon, has been reported by several authors after LVAD implantation and secondary removal, thereby avoiding heart transplantation. Perhaps the future of the Batista procedure will be linked to heterotopic heart transplantation, which will allow a temporary unloading of the repaired ventricle and thus safely differentiate between those patients able to live without a second heart and those who permanently need this assistance.

Heart failure surgery is at its early phase, especially for palliative procedures, and further elaboration and investigations are required before any definitive conclusions can be drawn.

GILLES DREYFUS SHERBAN MIHEALAINU

Université Paris $V$-René Déscartes,

Hôpital Foch,

Service de Chirurgie Cardio-Vasculaire,

40 rue Worth,

92151 Suresnes Cedex,

France

g.dreyfus@hopital-foch.org

1 Dreyfus GD, Duboc D, Blasco, et al. Myocardial viability assessment in ischemic cardiomyopathy: benefits of coronary revascularisation. Ann Thorac Surg 1994;57:1402-8.

2 Di Carli MF, Maddahi J, Rokhsar S, et al. Long-term survival of patients with coronary disease and left ventricular dysfunction: implications for the role of myocardial viability assessment in management decisions. $\mathcal{F}$ Thorac Cardiovasc Surg 1998;116:997-1004.

3 Dor V, Sabatier M, Di Donato M, et al. Efficacy of endoventricular patch plasty in large post infraction akinetic scar and severe left ventricle dysfunction: comparison with a series of large dyskinetic sears. $\mathcal{F}$ Thorac Cardiovasc Surg 1998;116:50-9.

4 Bolling SF, Pagani FD, Deeb GM, et al. Intermediate-term outcome of mitral reconstruction in cardiomyopathy. $\mathcal{f}$ Thorac Cardiovasc Surg 1998;115:381-8.

5 McCarthy JF, McCarthy PM, Starling RC, et al. Partial left ventriculectomy and mitral valve repair for end-stage congestive heart failure. Eur f Cardiothorac Surg 1998;13:337-43. 


\section{Editorial}

\section{Blood pressure measurement is changing!}

As we move into the new millennium, the century old Riva-Rocci/Korotkoff technique of measuring blood pressure is changing. There are a number of reasons for this. First, mercury is a toxic substance, the use of which can no longer be countenanced in clinical medicine, and the traditional technique, despite a history of reputable service, is likely to disappear from clinical practice. ${ }^{12}$ Second, it is now recognised that though the old technique has given good service, it is fraught with inaccuracies, ${ }^{3}$ and accurate automated devices are becoming available to replace the mercury sphygmomanometer. Third, 24 hour ambulatory blood pressure measurement (ABPM) has highlighted the phenomenon of white coat hypertension, and more reliance is being placed on blood pressure behaviour than on casual measurement of blood pressure levels. ${ }^{4}$

\section{Banning mercury}

Mercury is a toxic, persistent, and bioaccumable substance, many tons of which are distributed throughout the world to hospitals and countless individual doctors and little of which is returned for disposal. Admittedly the contribution of mercury from sphygmomanometers to environmental pollution is small, but none the less, mercury from whatever source finds its way back into the environment through evaporation, in sewage or in solid waste, most seriously damaging the marine environment, and it accumulates in soil and in sediments thereby entering the food chain. ${ }^{1}$ The mercury thermometer has been replaced in many countries, and in Sweden and the Netherlands the use of mercury is no longer permitted in hospitals. In other European countries, however, including the UK and Ireland, the move to ban mercury from hospital use has not been received with enthusiasm because we do not have an accurate alternative to the mercury sphygmomanometer. ${ }^{12}$ This ambivalence often results in hospitals and doctors replacing mercury sphygmomanometers with unreliable and inaccurate devices, such as aneroid sphygmomanometers, which become inaccurate with use and should not, therefore, be substituted for the mercury instrument. ${ }^{2}$ Many automated devices have had a poor record for accuracy, but automated devices are now beginning to satisfy the stringent criteria of the validation protocols of the British Hypertension Society (BHS) and the Association for the Advancement of Medical Instrumentation (AAMI). ${ }^{5}$

The passing of mercury sphygmomanometers should not in itself be a cause for concern. In fact, it might be argued that the sooner we rid ourselves of an inaccurate technique, on which we base so many important decisions of management, the better. ${ }^{13}$ Automated devices can remove observer error and provide in addition a printout of the measurement with the date and time of the measurement, or the measurement can be stored for display in a computer program.

Banning mercury from clinical use raises another issue of importance for clinical medicine. The Systeme International (SI) unit for pressure is the kilopascal. However, replacing the millimetre of mercury by the kilopascal has been postponed until such time as there is a suitable alternative to the mercury sphygmomanometer. ${ }^{6}$ If the millimetre of mercury is no longer the unit of measurement for blood pressure, the mainstay of the medical argument for retaining it as a unit of measurement-namely, that we measure what we see-will also disappear.

\section{White coat hypertension}

The importance of white coat hypertension rests on a curious haemodynamic phenomenon, which has quite profound clinical relevance: patients-let us call them people, because they may not be ill-who appear to have hypertension when their blood pressure is measured by the traditional Riva-Rocci/Korotkoff method, have normal blood pressures when ambulatory techniques are used to record their blood pressures away from the medical environment. Put another way, conventional blood pressure measurement is misleading in people with white coat hypertension (and most of us have some degree of white coat reaction), and if decisions are based on these measurements inappropriate diagnosis and treatment may result.

The best way to diagnose white coat hypertension is with ambulatory blood pressure measurement. ${ }^{7}$ The evidence from self measurement of blood pressure is not as conclusive, but the phenomenon can be demonstrated if home recorded blood pressures are normal with elevated clinic blood pressures. Unfortunately, there is no means of identifying people with white coat hypertension except by demonstrating the presence of normal blood pressures away from the medical environment.

The most popular definition is that blood pressure measured by conventional techniques in the office, clinic or surgery is above $140 / 90 \mathrm{~mm} \mathrm{Hg}$, but when ambulatory measurement is performed the blood pressures are normal throughout the 24 hour period, except perhaps during the first hour of the 24 hour recording when the patient is under the pressor influence of the medical environment while having the monitor fitted (fig 1). ${ }^{7}$ White coat hypertension is common, being present in about a quarter of people who appear to have hypertension with conventional measurement. ${ }^{8}$

The consequences of failing to identify white coat hypertension are considerable. Young (and indeed the not so young) people may be penalised for insurance and pension policies, and for employment. Life long treatment may be prescribed unnecessarily, and if antihypertensive medication is given to people whose 24 hour pressures are normal they may be made unwell by the adverse effects of medication. In the elderly, in whom white coat hypertension is common, the inappropriate use of drugs may have serious debilitating consequences.

Are people with white coat hypertension at risk? This is an important question because future management will be dependent on it. The evidence from a growing literature on the subject strongly supports the view that though white coat hypertension may not be altogether benign (some $10 \%$ of subjects show echocardiographic evidence of increased left ventricular hypertrophy), the risk of 


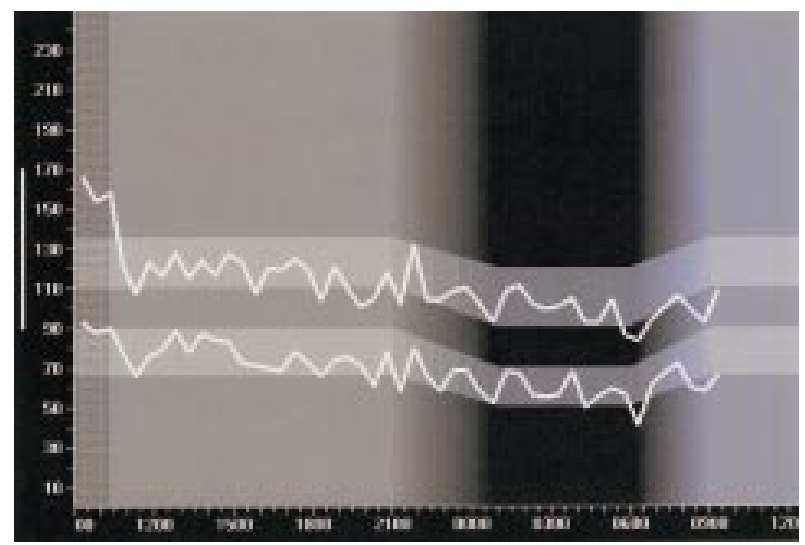

Figure 1 DABL cardiovascular plot. ${ }^{5}$ Vertical axis: blood pressure level; horizontal axis: time of day; hatched area: first hour of recording or "white coat window"; darkened area: night-time period; grey bands: limits of normality for systolic and diastolic blood pressures. ABPM plot shows white coat hypertension: office blood pressure 170/90 mm Hg (vertical bar); first hour blood pressure reaches $166 / 94 \mathrm{~mm} \mathrm{Hg}$, but then settles to give daytime pressures averaging $122 / 78 \mathrm{~mm} \mathrm{Hg}$ and night-time pressures averaging $112 / 68 \mathrm{~mm} \mathrm{Hg}$.

cardiovascular complications is very much less than for patients with sustained hypertension. ${ }^{9}$ Therefore, most people with white coat hypertension are not in need of antihypertensive medication, but because some may be at risk, albeit it considerably less so than patients with sustained hypertension, follow up is required. Of course, concomitant risk factors, such as smoking, obesity, and hypercholesterolaemia, should be addressed.

\section{Ambulatory blood pressure measurement}

It is recommended that people with white coat hypertension should be followed at yearly intervals with ambulatory measurement. ${ }^{10}$ In advocating the use of ambulatory measurement annually to follow people with white coat hypertension, the fiscal argument is often invoked, namely that the technique is too expensive to justify such a recommendation, and that indiscriminate use of ABPM would place an intolerable burden on health care services. Studies on the economics of ABPM are few, and the fear of inappropriate use, especially in private practice, has had a negative influence. ${ }^{4}$ However, the main riposte to the financial argument must be that if drug treatment is postponed or averted, the savings in pharmacological costs far outweigh the technologic costs of using ABPM. Until recently the evidence that ABPM might beneficially influence drug prescribing was lacking. However, in a study in which treatment was prescribed on the basis of either conventional blood pressure or mean daytime ABPM, significantly less antihypertensive medication was prescribed when ABPM was used, multiple drugs were used less frequently, and importantly there was no difference in left ventricular size between the two groups, showing that the patients for whom less medication was prescribed were not disadvantaged in terms of target organ status. ${ }^{11}$

The issue of equipment accuracy is very much better for ABPM devices than for other automated devices, with a large number of devices fulfilling the criteria of the BHS and AAMI protocols (for a list of devices see O'Brien and colleagues $^{10}$ ).

Another argument put forward against the use of ABPM is that there are not sufficient data from longitudinal studies showing its superiority over conventional measurement to justify its use in clinical practice. In fact this line of reasoning is no longer valid as the evidence is now available to show that $A B P M$ is superior to conventional measurement in predicting prognosis. ${ }^{12}$ However, when ABPM is used to find those people with elevated conventional blood pressure in whom blood pressure elevation is not sustained when they are removed from the pressor effect of the medical environment, the technique is merely a facet of good clinical practice in which the issue of outcome is secondary.

Who should have ABPM? This interesting question is addressed-but not answered satisfactorily-in three prestigious international guidelines on hypertension: the Joint National Committee on prevention, detection, detection, evaluation, and treatment on high blood pressure (JNC VI) ${ }^{13}$ recommends that ABPM should be performed in "suspected white coat hypertension", whereas the BHS, ${ }^{14}$ and the World Health Organization/ International Society of Hypertension (WHO/ISH) ${ }^{15}$ guidelines both recommend that ABPM should be performed when blood pressure shows "unusual variability". However, the guidelines do not say when white coat hypertension should be suspected, or what exactly constitutes unusual variability of blood pressure, simply because there are no identifying characteristics for white coat hypertension other than by demonstrating that elevation of blood pressure using conventional measurement is absent on ABPM. ${ }^{16}$ The BHS recommendations on the use and interpretation of ambulatory blood pressure measurement recommends the technique for the exclusion of white coat hypertension. ${ }^{10}$ But as every patient with apparent elevation of blood pressure in our clinics and surgeries could have white coat hypertension, does this mean that all such people should have ABPM? The answer has to be "yes" in patients with mild, borderline and moderate elevation of blood pressure on conventional measurement. I would not permit a doctor to prescribe life long treatment for me on the basis of conventional measurement, and this is also my recommendation for my patients. The benefits of identifying white coat hypertension are such that ABPM should be available to patients wherever and whenever the diagnosis of "hypertension" is contemplated.

\section{Self blood pressure measurement}

Self blood pressure measurement is re-establishing a place for itself in the management of hypertension. The First International Consensus Conference on Blood Pressure Self-Measurement was held in Versailles in June 1999. ${ }^{17}$ A large number of issues were examined and it was agreed that self measurement had a role in the management of hypertension, but that data were needed from longitudinal studies before its place could be firmly established. The technique should be seen as complementary to ABPM rather than a substitute for it, as both techniques allow blood pressure behaviour outside the medical environment to be assessed in different ways.

\section{EOIN O'BRIEN}

Blood Pressure Unit,

Beaumont Hospital,

Dublin 9, Ireland

eobrien@iol.ie

\footnotetext{
1 O'Brien E. Replacing the mercury sphygmomanometer. BMF 2000;320:815-16.

2 O'Brien E. Will mercury manometers soon be obsolete? f Hum Hypertens $1995 ; 9: 933-4$

3 Markandu ND, Whitcher F, Arnold A, et al. The mercury sphygmomanometer should be abandoned before it is proscribed. F Human Hypertens 2000; 14:31-6.

4 O'Brien E. White coat hypertension: how should it be diagnosed? $\mathcal{F}$ Human Hyperten 1999;13:801-2.

5 O'Brien E, Mee F, Atkins $\mathrm{N}$, et al. Evaluation of three devices for self-measurement of blood pressure: according to the revised British Hypertension Society protocol: the Omron HEM-705CP, Phillips HP5332, and Nissei DS-175. Blood Pressure Monitoring 1996; 1:55-61.

6 O'Brien E. Will the millimetre of mercury be replaced by the kilopascal. $f$ Hypertens 1998;16:259-61.
} 
7 Owens P, Atkins N, O'Brien E. The diagnosis of white coat hypertension by ambulatory blood pressure measurement. Hypertension 1998;34:267-72.

Pickering TG, James GD, Boddie C, et al. How common is white coat hypertension? $\mathcal{f} A M A \quad 1988 ; 259: 225-8$

9 Owens P, Lyons S, Rodriquez S, et al. Is elevation of clinic blood pressure in patients with white coat hypertension who have normal ambulatory blood pressure associated with target organ damage? $\mathcal{f}$ Hum Hypertens 1998;12:743-8

10 O'Brien E, Coats A, Owens P, et al. Use and interpretation of ambulatory blood pressure monitoring: British Hypertension Society recommendations. BMF 2000;320:1128-34.

11 Staessen JA, Byttebier G, Buntinx F, et al, for the Ambulatory Blood Pressure Monitoring and Treatment of Hypertension Investigators. Antihypertensive treatment based on conventional or ambulatory blood pressure measurement. A randomized controlled trial. $7 A M A$ 1997;278: 1065-72.

12 Verdecchia P. Prognostic value of ambulatory blood pressure current evidence and clinical implications. Hypertension 2000;35:844-51.
13 The Joint National Committee of Prevention, Detection, Evaluation, and Treatment of High Blood Pressure. The sixth report of the Joint National Committee on prevention, detection, evaluation and treatment of high

14 Ramsay LE, Williams B, Johnston GD, et al. Guidelines for management of hypertension: report of the third working party of the British Hypertension Society. F Human Hypertens 1999;13:569-92.

15 Guidelines Subcommittee of the World Health Organisation-International Society of Hypertension (WHO-ISH). 1999 World Health OrganisationInternational Society of Hypertension guidelines for the management of hypertension. F Hypertens 1999;17:151-83.

16 O'Brien E, Staessen J. Critical appraisal of the JNC VI, WHO/ISH and BHS guidelines for essential hypertension. Expert Opinion on Pharmacotherapy and Emerging Drugs 2000;1:675-82.

17 Asmar R, Zanchetti A on behalf of the Organizing Committee and participants. Guidelines for the use of self-blood pressure monitoring: a summary pants. Guidelines for the use of self-blood pressure monitoring. a summary Hypertension 2000;18:493-508.

\section{IMAGES IN CARDIOLOGY}

\section{Aortic valve sparing operations}

A new aortic Dacron prosthesis (Sulzer Vascutek, Renfrewshire, UK) that replaces the aortic root and at the same time recreates the sinuses of Valsalva has been designed (top; LV, left ventricle). It has been employed in the two types of surgical techniques more commonly used to spare the aortic valve: the reimplantation (or David I) technique where the natural valve is sutured inside the Dacron conduit, and the remodelling (or Yacoub) technique where the Dacron conduit is tailored to fit the crescent shape of the aortic root.

A transoesophageal echocardiographic long axis view of the aortic root during diastole in a patient after a Yacoub type of valve sparing procedure (middle; Ao, prosthetic ascending aorta) and during systole in a patient after a David I type of valve sparing procedure using the new Dacron conduit (bottom) shows the normal shape and dimension of the sinuses, the natural narrowing of the sinotubular junction, and the perfect alignment of the open leaflet with the prosthetic ascending aortic wall.

Upon implantation of the new aortic root conduit and without technical modification of the techniques as originally described by their authors, it is possible to restore a perfect anatomy of the aortic root.

RUGGERO DE PAULIS GIOVANNI MARIA DE MATTEIS LUIGI CHIARIELLO

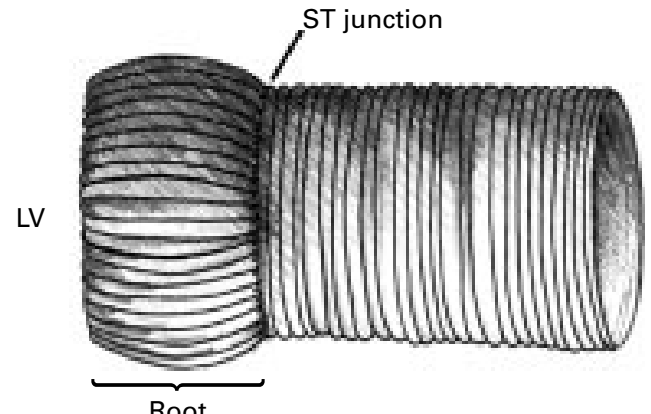

Root
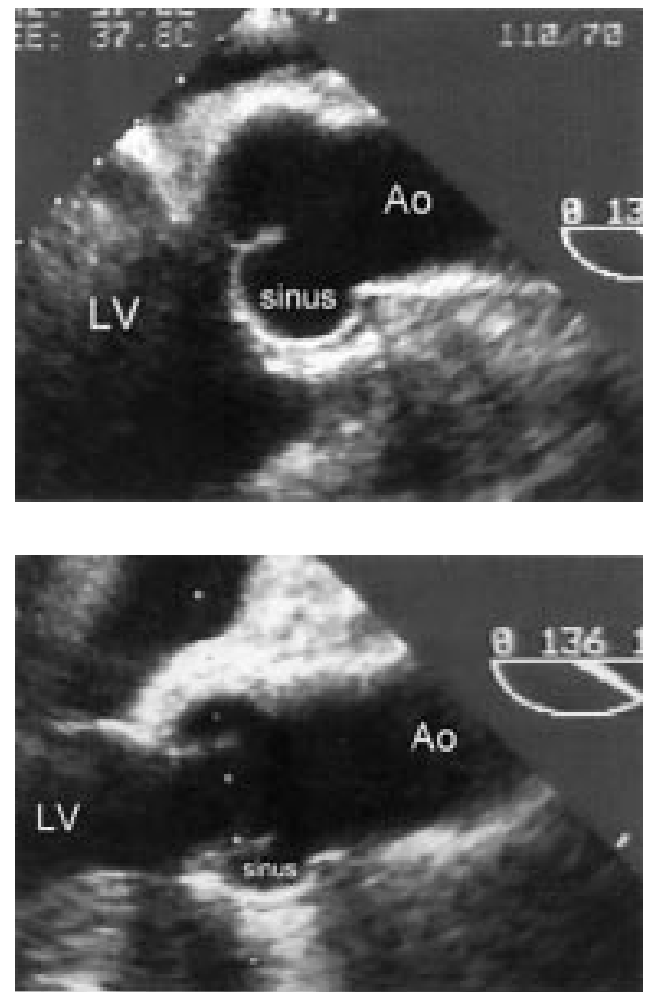


\section{Managing out-of-hospital cardiac arrest survivors: 1. Neurological perspective}

Survival from out-of-hospital cardiac arrest is becoming an increasingly common occurrence, because of defibrillation initiatives and increased public awareness of basic life support skills. ${ }^{1-4}$ Two main factors determine survival from an out-of-hospital cardiac arrest: prompt administration of effective cardiopulmonary resuscitation (CPR); and early defibrillation. In addition to increasing the number of victims who survive to discharge, these interventions also allow some individuals to survive who would have otherwise succumbed immediately, only for them to die later because of the sequelae of cerebral hypoxia. Thus, there is an increasing population of cardiac arrest victims who survive with neurological injury. For those who avoid lethal brain injury, the initial priority is the assessment of the risk of further arrhythmic events. This is important because treatments such as revascularisation, antiarrhythmic drugs, and implantable cardioverter defibrillators (ICDs) reduce the risk of subsequent death in some subgroups. Resuscitated cardiac arrest victims present a challenge on two fronts: assessment and treatment of neurological injury, and assessment and intervention to minimise risk of further arrhythmic events. This first article focuses on early management and neurological sequelae of cardiac arrest. The second article in this series focuses on investigating the substrate for cardiac arrest, and interventions that reduce the risk of further arrhythmic events.

\section{Immediate management}

After admission, resuscitated cardiac arrest victims may be unstable for several reasons. Nearly half require mechanical ventilation, and aspiration of gastric contents may exacerbate respiratory compromise. Peri-arrest arrhythmias are common, and management guidelines are published by the Resuscitation Council (UK). ${ }^{5}$ Hypotension can result from the process that leads to the cardiac arrest (for example, myocardial infarction) and from post-resuscitation myocardial stunning, necessitating the use of inotropes or mechanical circulatory support, or both. Many resuscitated cardiac arrest victims are elderly and have serious underlying comorbidity. Essential to their management is involvement of their family at this stage in discussion about treatment. In this way, patients can be managed at a level appropriate to their overall prognosis and quality of life. Age alone should not dictate whether or not intensive treatment is given, since the elderly have almost as good a prospect of survival to discharge as younger patients after initially successful resuscitation. ${ }^{6}$

Management of specific triggering pathologies (for example, acute myocardial infarction) will be discussed in the second article in this series.

\section{Mechanisms of brain injury during and after cardiac arrest}

Data from animal models, and limited human studies of neonatal hypoxia and cerebral hypoperfusion during cardiopulmonary bypass, indicate that brain injury occurs through several mechanisms. Hypoxia itself is the main factor-failure of ATP dependent cell membrane ion transporters leads to influx of calcium into the neuronal cytoplasm, causing cell death. In addition, oxidative stress occurs after resuscitation due to abrupt restoration of tissue blood flow and administration of supraphysiological concentrations of oxygen. ${ }^{7}$ Free radical mediated tissue injury occurs, leading to microvascular damage and impaired reflow, similar to that seen after coronary reperfusion. ${ }^{89}$ Cerebral oedema may follow, caused by movement of fluid from the intravascular compartment into the hyperosmotic interstitial space. ${ }^{10}$ Infiltration of tissue by neutrophils may result from release of chemotactic factors and expression of integrins (for example, CD11b/ CD18 and ICAM-1), and further tissue injury may occur. ${ }^{11}$ Finally, neuronal injury may also result from cerebral hypoperfusion after resuscitation caused by reduction in cardiac output. "Watershed" zones (for example, the hippocampus) may be especially prone to injury during and after resuscitation because of their relatively poor blood supply, potentially leading to memory and visiospatial deficits.

\section{Assessment and management of hypoxic brain injury}

Accurate prognostic assessment of cardiac arrest survivors is important because treatment decisions are partly governed by the patient's prognosis. If a given patient is assessed as having zero probability of survival to discharge, treatments such as CPR, antibiotic therapy or organ support may be inappropriate. Conversely, it is important not to deprive patients of these treatments if their prospect of survival is real. Knowledge about the patient's prognosis also helps when counselling their family about the likely outcome.

SPECIFICITY: THE KEY TO PROGNOSTIC ASSESSMENT

Many cardiac arrest victims are admitted to hospital unconscious, with an uncertain prognosis, and are difficult to assess and manage. More than $75 \%$ of initially comatose patients die during hospital admission. ${ }^{12}$ When assessing a patient's prognosis, an important issue is the specificity of the tests used. In other words, it is important for that test to be as certain as possible when it predicts in-hospital death, otherwise the patient could be denied treatment when their prospect of survival is not hopeless. Sensitivity is less important; no prognostic test can identify all patients who will die in hospital, because death occurs from many causes-cerebral hypoxia, arrhythmias, pump failure, sepsis, etc. Many methods have been evaluated for assessing the prognosis of cardiac arrest victims. These include clinical algorithms, neurophysiological tests, and tests which quantify structural brain injury.

CLINICAL METHODS

Clinical assessment aids risk stratification of cardiac arrest victims. The Glasgow coma score (GCS) has been 

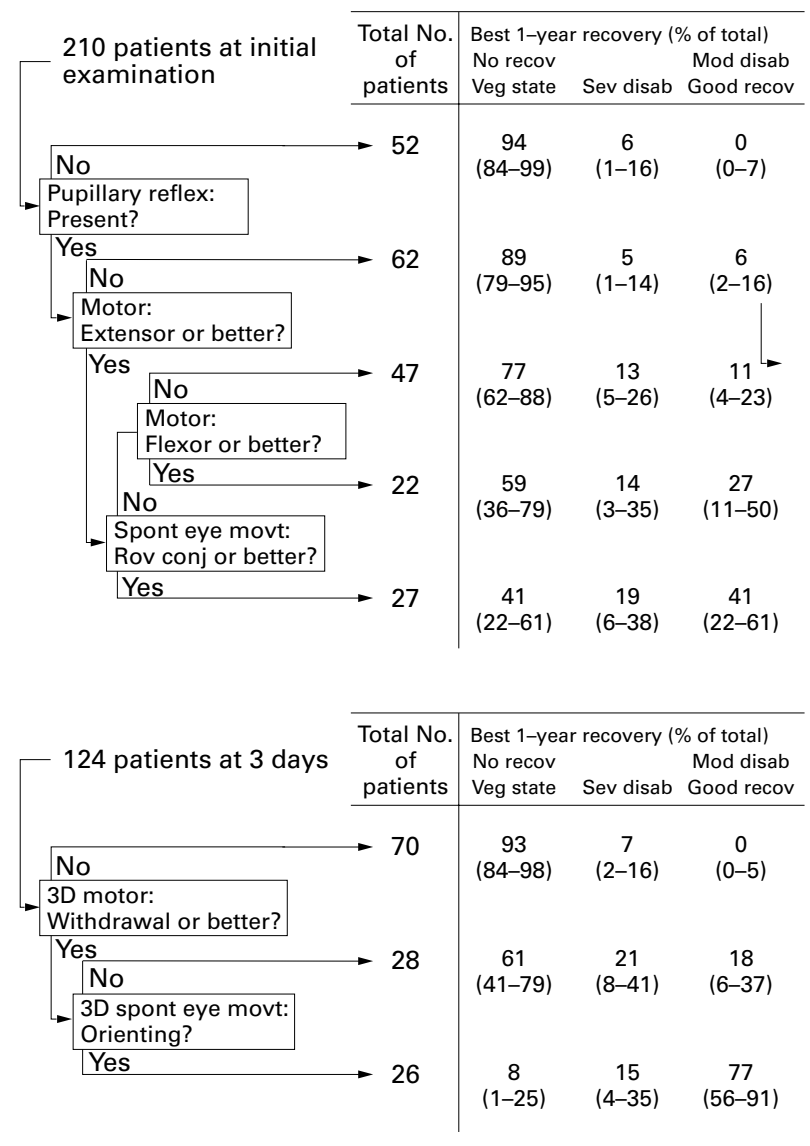
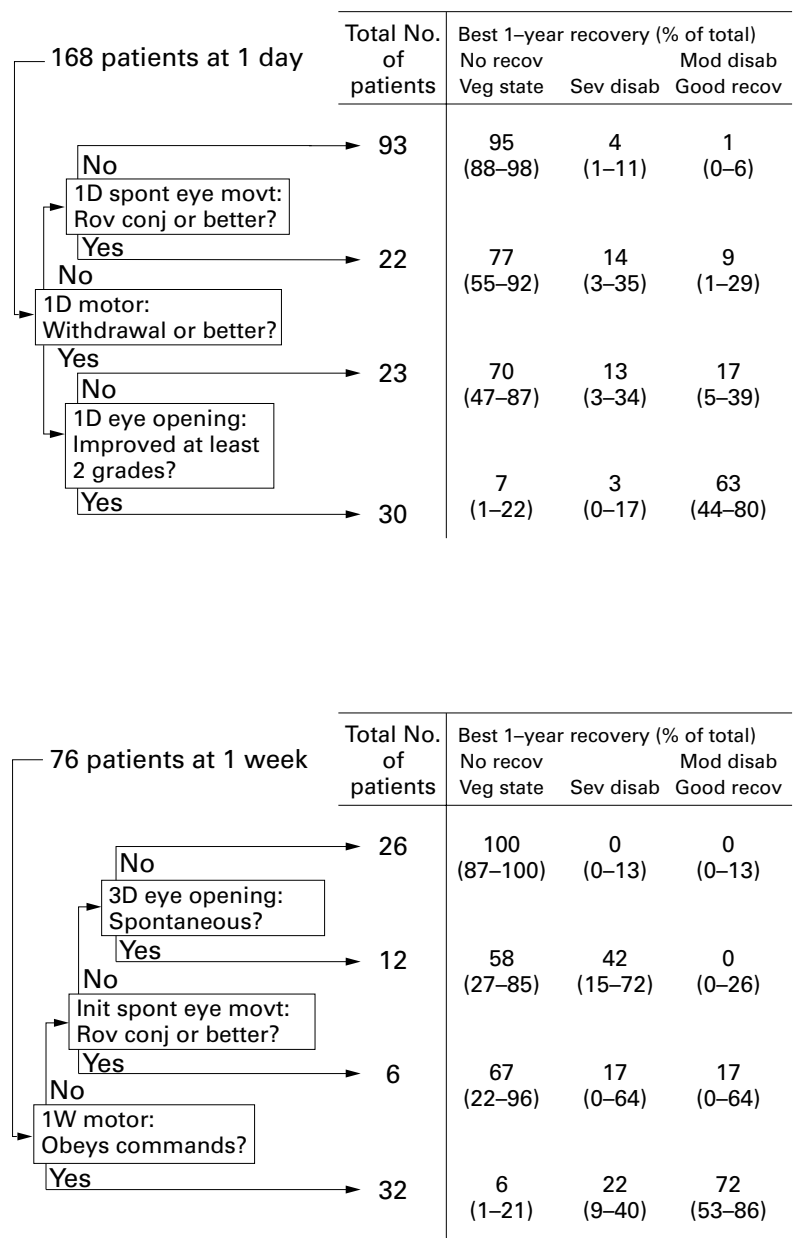

Figure 1 Prediction of outcome from hypoxic-ischaemic coma using clinical criteria. Patients are classified according to best functional status within the first year. Figures in brackets are 95\% confidence intervals for the percentages cited. Reproduced with permission from Levy et al. $\mathcal{F} A M A$

1985;253:1420-6. Copyrighted 1985, American Medical Association

evaluated in several studies. ${ }^{12}$ Although a low admission GCS identifies patients at greatest risk of in-hospital death, in most studies high specificity is only achieved when the GCS is measured three to five days after admission. Even then, patients with an intermediate GCS have an indeterminate prognosis. Its utility is limited in ventilated patients and in patients treated with sedation and neuromuscular blockade. A more sophisticated scoring system, incorporating variables such as pupil response, doll's eyes reflex, blink reflex, and presence or absence of seizures, was developed by Levy, based on data from more than 200 patients with hypoxic-ischaemic coma. From these data a flow chart algorithm has been developed to predict prognosis at different time points after cardiac arrest (fig 1). ${ }^{13}$ In particular, persistent absence of the pupillary reflex predicted either death or severe neurological disability with absolute specificity. This type of algorithm is useful, and requires consistency in examination technique, but it is also of limited use in patients receiving sedation or neuromuscular blockade.

NEUROPHYSIOLOGICAL TESTING

The two main techniques for neurophysiological assessment are electroencephalography (EEG), which measures cerebral electrical activity at rest, and cerebral evoked potentials, measured in response to defined stimuli. Specific EEG findings, such as the alpha coma, burst suppression, and isoelectric patterns, have been identified with an adverse prognosis. ${ }^{14}$ These lack specificity, with some patients surviving despite their presence. In contrast cerebral evoked potentials, such as the cortical response to median nerve stimulation, can reliably identify subgroups with a poor prognosis with a specificity that is superior to clinical algorithms and to the EEG.

QUANTITATION OF BRAIN INJURY

Clinical and neurophysiological techniques rely on assessing brain function. An alternative approach is to estimate the magnitude of the brain injury caused by cardiac arrest. Magnetic resonance imaging and computerised tomography are of limited use, and can usually only detect gross abnormalities such as watershed infarcts or intracranial haemorrhage. Brain injury can also be measured using biochemical markers in a manner analogous to that of cardiac enzymes in myocardial infarction. Two markers appear promising from recent trials-protein S-100 (a glial protein), and neuron-specific enolase (NSE). One early report indicated that a serum NSE concentration greater than $33 \mu \mathrm{g} / 1$ measured within a week of cardiac arrest had a sensitivity of $80 \%$ and a specificity of $100 \%$ for persistent coma. ${ }^{15}$ Estimation of serum S-100 concentration as early as 24 hours after cardiac arrest identifies a subgroup with an apparently hopeless prognosis (S-100 concentration $\geqslant 0.7 \mu \mathrm{g} / \mathrm{l}) .{ }^{16}$ These findings are based on small cohorts and require confirmation in a larger scale study before they can be applied to clinical decision making.

DO ADJUNCTIVE TREATMENTS IMPROVE PROGNOSIS?

Several adjunctive treatments have been evaluated in attempts to limit brain injury after cardiac arrest. These 


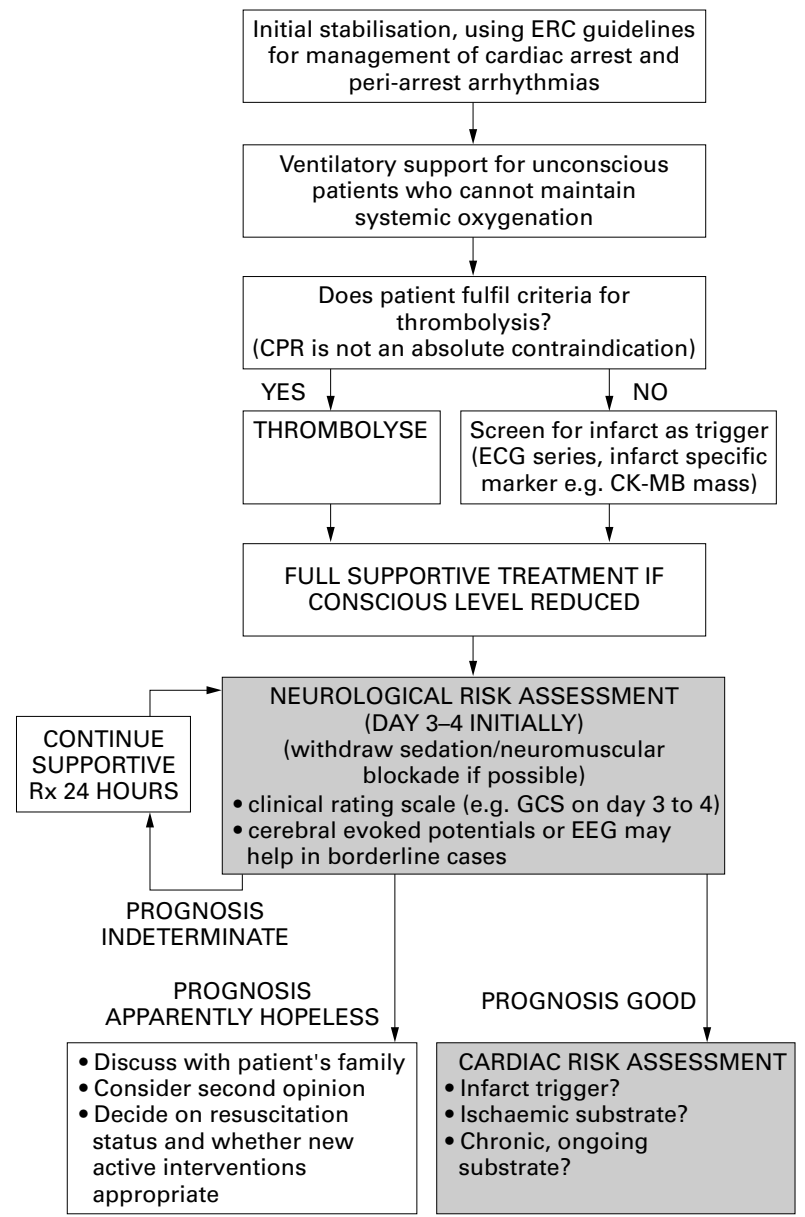

Figure 2 Suggested algorithm for early management of out-of-hospital cardiac arrest survivors.

include administration of glucose (as a substrate for brain metabolism), dexamethasone and mannitol (to reduce osmotic load and cerebral oedema), barbiturates (to reduce cerebral oxygen demand), and nimodipine (to improve cerebral blood flow and to reduce entry of calcium into neurons). None of these agents has been conclusively shown to improve outcome. Preliminary data suggest that induction of mild hypothermia during cardiac arrest affords a degree of cerebral protection, but it is difficult to envisage how this could be practically applied. ${ }^{17}$ Perhaps the most encouraging development in neuroprotection relates to experimental evidence of limitation of brain injury with antioxidant agents, notably the 21aminosteroid tirilazad. ${ }^{18}$ Since oxidative stress can persist for several hours after cardiac arrest, such agents may in future have a role in neuroprotection.

\section{QUALITY OF SURVIVAL: IS RESUSCITATION WORTH THE} EFFORT?

Cardiac arrest victims may survive after a prolonged but non-lethal period of cerebral hypoxia. The resulting neurological injury can be severe enough to affect cognitive function and to compromise patients' independence. Overt neurological deficits are surprisingly uncommon, affecting less than $5 \%$ of patients. Cognitive dysfunction is more prevalent, affecting between $20-50 \%$ of patients. ${ }^{19}$ Memory impairment is common and may affect the patient's ability to return to work and to perform daily activities. Despite this, most out-of-hospital cardiac arrest survivors are functionally independent and have intact cognitive function.

\section{Conclusions}

Cardiologists and intensivists can expect to care for a growing population of out-of-hospital cardiac arrest survivors. Most are unconscious on admission to hospital. Although early awakening is associated with a good neurological prognosis, the prognosis of those who remain comatose is difficult to assess, and management decisions are difficult. Clinical prognostic scoring systems and cortical evoked potentials help in their evaluation, and can assist when planning counselling of relatives. Figure 2 summarises the key elements of early management. There is a need to develop more reliable methods of prognostic assessment that are specific at identifying patients who have a hopeless prognosis at an early stage, ideally on the first day of admission. For those who survive, identification of the cause of cardiac arrest is central to assessment of future risk and in planning their management.

University of Edinburgh Cardiovascular Unit,

NEIL R GRUBB

Cardiology Department,

Royal Infirmary,

1 Lauriston Place,

Edinburgh EH3 9YW, UK

N.Grubb@ed.ac.uk.

1 Cobb LA, Werner JA, Trobaugh GB. Sudden cardiac death: 1. A decade's experience with out-of-hospital resuscitation. Mod Concepts Cardiovasc Dis 1980;49:31-6.

2 Guzy PM, Pearce ML, Greenfield S. The survival benefit of bystander cardiopulmonary resuscitation in a paramedic-served metropolitan area. $\mathrm{Am} \mathcal{F}$ Public Health 1983;73:766-9.

3 Cobbe SM, Redmond MJ, Watson JM, et al. "Heartstart Scotland"-initial experience of a national scheme for out of hospital defibrillation. BMF 1991;302:1517-20.

4 Dickey W, McKenzie G, Adgey AAJ. Long-term survival after resuscitation from ventricular fibrillation occurring before hospital admission. $Q \mathcal{F M}$ 1991;80:729-37.

5 Chamberlain D. Peri-arrest arrhythmias. Br f Anaesthesia 1997;79:198-202.

6 Juchems R, Wahlig G, Frese W. Influence of age on the survival rate of outof-hospital and in-hospital resuscitation. Resuscitation 1993;26:23-9.

7 Palmer C. Hypoxic-ischaemic encephalopathy, therapeutic approaches Palmer C. Hypoxic-ischaemic encephalopathy, therapeutic approaches
against microvascular injury, and role of neutrophils, PAF and free radicals.
Clinics Perinatol 195;22:481-517.

8 White BC, Gross LI, O'Neill BJ, et al. Global brain injury and reperfusion. Ann Emerg Med 1996;27:588-94.

9 Bottiger BW, Krumniki JJ, Gass P, et al. The cerebral 'no-reflow' phenomenon after cardiac arrest in rats-influence of low flow reperfusion. Resuscitation 1997;34:79-87.

10 Jannucci RC, Christensen MA, Yagen JY. Nature, time course and extent of cerebral edema in perinatal hypoxic-ischemic brain damage. Paed Neurol 1993;9:29-34

11 Howard EF, Chen Q, Cheng C, et al. NF-kappa B is activated and ICAM-1 gene expression is upregulated during reoxygenation of human brain endothelial cells. Neurosci Letters 1998;248:199-203.

12 Verbruggen $\mathrm{H}$, Van den Brock L, Corne L, et al. Predictive value of Glasgow coma score for awakening after out-of-hospital cardiac arrest. Cerebral resuscitation study group of the Belgian Society for Intensive Care. Lancet resuscitation study

13 Levy DE, Caronna JJ, Singer $\mathrm{BH}$, et al. Predicting outcome from hypoxic-ischaemic coma. fAMA 1985;253:1420-6.

14 Zandbergen EGJ, de Haan RJ, Stoutenbeek CP, et al. Systematic review of early prediction of poor outcome in anoxic-ischaemic coma. Lancet 1998;352:1808-12.

15 Barone FC, Clark RK, Price WJ, et al. Neuron-specific enolase increases in cerebral and systemic circulation following focal ischaemia. Brain Research 1993;623:77-82

16 Martens P, Raabe A, Johnsson P. Serum S-100 and neuron specific enolase for prediction of regaining consciousness after global cerebral ischaemia. Stroke 1998;29:2363-6.

17 Yanagawa Y, Ishihara S, Norio H, et al. Preliminary clinical outcome study of mild resuscitative hypothermia after out-of-hospital cardiac arrest. Resuscitation 1998;39:6-66.

18 Sterz F, Janata K, Kurciyan I, et al. Possibilities of brain protection with tirilazad after cardiac arrest. Seminars Thrombosis Haemostasis 1996;22:10512.

19 Grubb NR, O'Carroll R, Cobbe SM, et al. Chronic memory impairment after cardiac arrest outside hospital. BMF 1996;313:143-6. 


\section{Editorial}

\section{Should we give antibiotic prophylaxis against infective endocarditis in all cardiac patients, whatever the type of dental treatment?}

The findings of Al-Karaawi and colleagues in the present issue are disturbing. ${ }^{1}$ One may conclude from their work that antibiotic prophylaxis against infective endocarditis should be applied more largely for dental procedures for which guidelines usually do not recommend such prophylaxis. At the same time, the usefulness of antibiotic prophylaxis is debated by many authors. ${ }^{23}$ Fortunately, prophylaxis is still recommended by authors who debate about it, and Al-Karaawi and colleagues do not draw the conclusion suggested above.

\section{Antibiotic prophylaxis and dental treatments: to be extended to more patients?}

In 136 children with severe congenital cardiac disease who underwent dental procedures, Al-Karaawi and colleagues calculated a cumulative exposure to bacteraemia over one year for several dental procedures, which were considered as prophylaxis or non-prophylaxis procedures according to the current US guidelines. ${ }^{4}$ They found that the cumulative exposure was as high or higher in many dental procedures for which prophylaxis is not recommended than for those for which prophylaxis is recommended. This was especially so for rubber dam placement.

The cumulative exposure was the product of the intensity of bacteraemia by the proportion of positive blood cultures by the length of bacteraemia by the number of times a given dental procedure was performed annually. This was a retrospective study, and since many data were not available in the files, the authors obtained the information from the literature, except for the number of times a given dental procedure was performed annually in a given child. It would have been much more interesting to use real data, and since most of the children were treated under general anaesthesia, at least in them drawing blood for cultures looks easy to do. Duration of bacteraemia was set at 15 minutes, whatever the type of dental procedure and whatever the child. Everybody who has to go to his or her dentist knows very well that the duration of a given dental procedure - and thus of bacteraemia - varies greatly in the same person according to the difficulty of the procedure! These are reasons why the results must be interpreted cautiously. The authors are aware of it, since the title clearly states that this is a theoretical analysis and since they draw very cautious conclusions: "The data raise important questions about the appropriateness of current guidelines for antibiotic prophylaxis of bacterial endocarditis." It looks timely to perform a prospective study, and to really measure all the components of cumulative exposure.

Unfortunately, some missing information would have been very useful. We know that the children had severe congenital cardiac disease, but we do not have any details about the types of cardiac diseases, although the risk of infective endocarditis depends on the underlying congenital heart disease. ${ }^{5}$ Not being dentists, and since the bacteraemia cannot occur without bleeding during the dental procedure, we wonder whether the bacteraemia after rubber dam placement is due to the rubber dam placement itself or to the work the dentist does before placing the rubber dam.

\section{Antibiotic prophylaxis and dental treatments: to be} restricted to fewer patients?

Numerous guidelines have been issued in many countries. Beside the British recommendations, ${ }^{6}$ the most recent ones come from USA ${ }^{4}$ and from a European group of experts. ${ }^{7}$ The US guidelines give a very precise list of dental procedures for which prophylaxis of infective endocarditis is recommended and for which it is not recommended ${ }^{4}$ - the same list which is given in the paper by Al-Karaawi and colleagues. ${ }^{1}$ In the European text-not cited by Al-Karaawi and colleagues - all dental procedures require prophylaxis in cardiac patients at risk, the only exceptions being procedures without risk of bleeding such as superficial caries and bloodless supragingival prosthetic preparations. ${ }^{7}$

In spite of numerous guidelines on prophylaxis of infective endocarditis, the incidence of infective endocarditis does not decrease over time. ${ }^{8}$ Several explanations for this apparent discrepancy can be proposed ${ }^{9}$ :

- compliance of physicians, dentists, and patients with guidelines is not good;

- prophylaxis of infective endocarditis is not efficacious;

- prophylaxis of infective endocarditis is efficacious, but its effect on the incidence of infective endocarditis is counterbalanced by the increasing frequency of patients at risk and of procedures at risk.

Efficacy of prophylaxis is not demonstrated, and it probably never will be. The only way to demonstrate efficacy would be a randomised trial, which appears unethical and not feasible; it has been calculated that over 6000 patients would be needed. ${ }^{10}$ Results from several case-control studies are conflicting - the protective efficacy of prophylaxis goes from less than $50 \%{ }^{11}{ }^{12}$ to more than $90 \%$. ${ }^{13}$ The link between dental procedures and infective endocarditis is not definitely established. ${ }^{14}$ Most of the data on efficacy of prophylaxis come from experimental infective endocarditis in animals, and this may not be extrapolated to mankind. ${ }^{15}$

Some apparent failures of prophylaxis have been described, $92 \%$ of which occurred after a dental procedure. ${ }^{16}$ But in the large majority of the cases, the prophylaxis did not perfectly conform to the American Heart Association recommendations.

In his editorial accompanying the Strom case-control study, ${ }^{14}$ Durack goes as far as advising prophylaxis only in cases of dental extraction or gingival surgery (including implant placement), only in patients with prosthetic valves or previous infective endocarditis. "As a first approximation, it should be possible to retain at least $80 \%$ of any putative benefits from currently recommended prophylaxis for less than $20 \%$ of the costs".

Finally?

A clear answer to the question, "Which patient, and for which dental treatment, should receive antibiotic prophylaxis against infective endocarditis?" does not exist yet, and 
much more work is needed in order to make clear the procedures at risk of infective endocarditis and to estimate the efficacy of prophylaxis.

FRANCCOIS DELAHAYE GUY DE GEVIGNEY

Hôpital Cardio-Vasculaire et Pneumologique,

BP Lyon Montchat,

69394 - Lyon Cedex 03,

France

francois.delahaye@chu-lyon.fr

1 Al-Karaawi ZM, Lucas VS, Gelbier M, et al. Dental procedures in children with severe congenital cardiac disease: a theoretical analysis of prophylaxis and non-prophylaxis procedures? Heart 2001;85:66-8.

2 Durack DT. Antibiotics for prevention of endocarditis during dentistry: time to scale back? Ann Intern Med 1998;129:829-31.

3 Wahl MJ. Myths of dental-induced endocarditis. Arch Intern Med 1994;154: 137-44.

4 Dajani AS, Taubert KA, Wilson W, et al. Prevention of bacterial endocarditis. Recommendations by the American Heart Association. Circulation 1997;96:358-66.

5 Corone P, Levy A, Hallali P, et al. A propos de 54 cas d'endocardites infectieuses observées en 32 ans sur une population de 2038 cardiopathies congénitales. Arch Mal Cœur 1989;82:779-84.
6 Working party of the British Society for Antimicrobial Chemotherapy. Antibiotic prophylaxis and infective endocarditis. Lancet 1992;339:1292-3.

7 Leport C, Horstkotte D, Burckhardt D, and the Group of Experts of the Leport C, Horstkotte D, Burckhardt D, and the Group of Experts of the International Society for Chemotherapy. Antibiotic prophylaxis for infective endocarditis from an international group of experts towards a European consensus. Eur Heart f 1995;16(suppl B):126-31

8 Delahaye F, Goulet V, Lacassin F, et al. Characteristics of infective endocarditis in France in 1991. A 1-year survey. Eur Heart F 1995;16:394-401.

9 Couturier F, Hansmann Y, Descampeaux C, et al. Les limites de l'antibioprophylaxie des endocardites infactieuses. Méd Mal Infect 2000;30: 3-10.

10 Durack DT. Prevention of infective endocarditis. $N$ Engl $f \mathrm{Med}$ 1995;332:38-44

11 Lacassin F, Hoen B, Leport C, et al. Procedures associated with infective endocarditis in adults. A case-control study. Eur Heart 7 1995;16:1968-74.

12 Van der Meer JTM, van Wijk W, Thompson J, et al. Efficacy of antibiotic prophylaxis for prevention of native-valve endocarditis. Lancet 1992;339: prophylax.

13 Imperiale TF, Horwitz RI. Does prophylaxis prevent postdental infective endocarditis? A controlled evaluation of protective efficacy. $\mathrm{Am} \mathcal{F} \mathrm{Med}$ 1990;88:131-6.

14 Strom BL, Abrutyn E, Berlin JA, et al. Dental and cardiac risk factors for infective endocarditis. A population-based, case-control study. Ann Intern Med 1998;129:761-9.

15 Francioli M, Francioli P, Glauser MP. Prophylaxie de l'endocardite bactérienne: bases expérimentales et conduite pratique. Rev Méd Suisse Romande 1988;108:665-70.

16 Durack DT, Kaplan EL, Bisno AL. Apparent failures of endocarditis prophylaxis. Analysis of 52 cases submitted to a national registry. $\mathscr{f} A M A$ 1983;250:2318-22.

\section{IMAGES IN CARDIOLOGY}

\section{"Late-late" reocclusion after coronary stenting and brachytherapy}

In November 1998, a 63 year old male patient with residual angina after a first myocardial infarction, was treated with coronary angioplasty and stenting for a critical stenosis of the mid-portion of the left anterior descending artery. He was included in the BRIE ( $\beta$ irradiation in Europe) trial and after dilatation, he received 14 grays of $\beta$ radiation from a $3 \mathrm{~cm}$ length source (Novoste) (below left). According to the protocol of the study at that moment, ticlopidine $250 \mathrm{mg}$ twice a day was prescribed for one month together with long life aspirin treatment.

In May 1999, at the planned six month follow up, control coronary angiography revealed no restenosis in the left anterior descending artery (below middle).

In August 1999 the patient developed a sudden episode of rest angina. Angiography revealed a subtotal occlusion in the stent (below right). He underwent a further balloon dilatation with good angiographic result and was discharged with ticlopidine $250 \mathrm{mg}$, twice a day, for six months and aspirin.
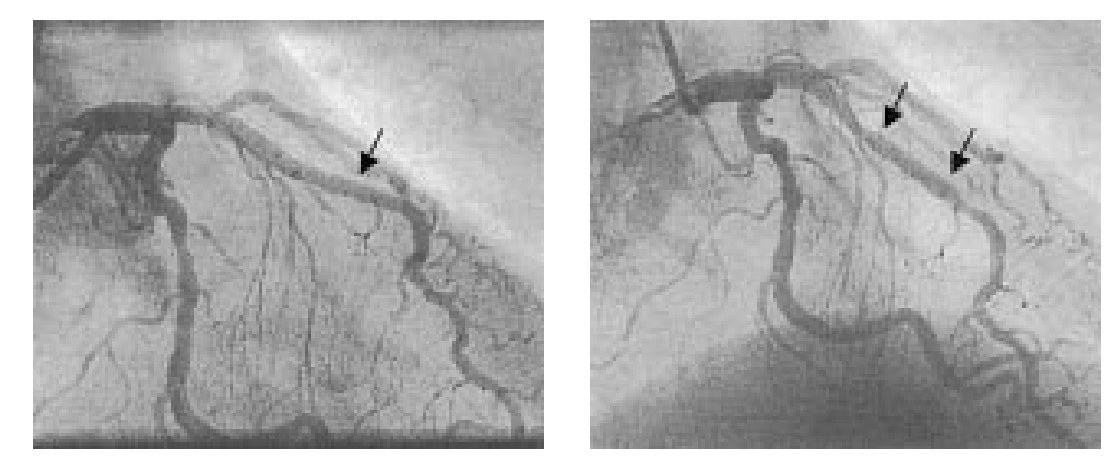

The problem of stent thrombosis more than six months after stent implantation is relatively new, and is associated with concomitant vascular brachytherapy. For patients with a patent treatment site at six months which subsequently develops a total occlusion, the term "late-late" thrombosis has been proposed (Waksman R. Circulation 1999;100:780-2). Potential causes include: (1) delayed re-endothelialisation; (2) fibrin deposition and platelet recruitment; (3) impaired vasoreactivity and spasm; (4) tissue erosion around the stent; and (5) unhealed dissections. The current judgement among most investigators is that prolonged antiplatelet treatment with aspirin plus ticlopidine or clopidogrel for one year after vascular brachytherapy will totally resolve this issue.

PETER VANDERGOTEN MARC BROSENS EDOUARD BENIT

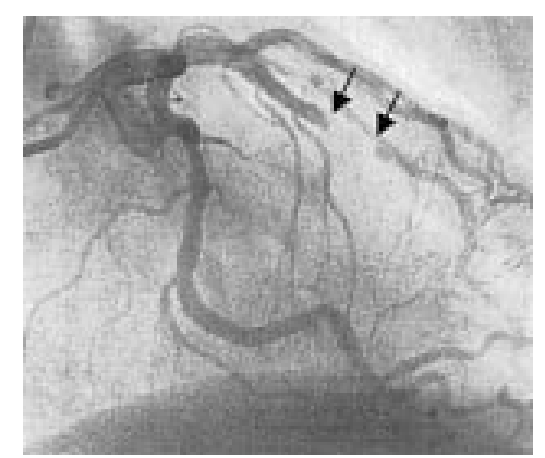

